\title{
A prediction of the onset of solar cycle 24
}

\author{
Z. L. Du \\ National Astronomical Observatories, Chinese Academy of Sciences, Beijing 100012, PR China \\ e-mail: zldu@bao.ac.cn \\ Received 11 January 2006 / Accepted 15 May 2006 \\ ABSTRACT

\begin{abstract}
Aims. This paper aims to search for a method to estimate the onset of a new solar cycle by re-investigating the relationship between descending and ascending phase durations in terms of weighted extremum times.

Methods. The lagged cross-correlation method is used to analyze their relationship.

Results. The descending phase durations are found to be anti-correlated (correlation coefficient, -0.77 ) with the ascending phase durations three cycles earlier, in the 13-month running mean sunspot numbers since cycle 8 . From linear regression analysis, the descending phase duration of cycle 23 is estimated to be $78 \pm 7$ months; thus, cycle 24 should start near March 2007, where the error bar equals \pm standard error.
\end{abstract}

Key words. Sun: activity - Sun: sunspots - Sun: general

\section{Introduction}

The maximum sunspot number $(\mathrm{SN}), R_{\mathrm{m}}$, and the times (or dates) of maxima and minima are important parameters describing solar activity cycles. The interval between successive maximum or minimum times defines the cycle lengths.

There are many methods of predicting $R_{\mathrm{m}}$; of these, the socalled precursor methods are believed to be superior to the socalled non-precursor methods, as regards accuracy (Ohl 1966; Kane 1978, 2001; Wilson 1990; Thompson 1993; Li 2001; Zhang \& Wang 1999; Du 2006a).

On the other hand, there are not so many methods of predicting the times of maxima or minima. One reason may be that there are no strong relationships between the cycle lengths and other cycle parameters. Although the correlation coefficient (CorrCoef) between the duration of the ascending phase $\left(T_{\mathrm{a}}\right)$ and $R_{\mathrm{m}}$ of the same cycle is quite high (CorrCoef $=-0.723$ for cycles 1-21, Wilson 1988), we cannot employ this correlation to accurately predict either of them because if one is unknown, then the other is also unknown.

There are several methods used to predict the extremum times of solar activity cycles. Gleissberg (1971), based on the idea of similar cycles (similar maxima have similar periods, see also Wang \& Han 2000; and Wang et al. 2002), estimated the period, $T_{\mathrm{a}}$ and descending phase duration $\left(T_{\mathrm{d}}\right)$. Wilson et al. (1998), based on the relationship between $R_{\mathrm{m}}$ and $T_{\mathrm{a}}$, predicted that cycle 24 will begin in January-October 2006, or before May 2007. Whitehouse (1985) predicted the extremum times by the modulation of phase variations in the differences between observed cycle lengths and the mean ones. Landscheidt (1999), based on the partition of the 178.8 year intervals between instances of retrograde motion in the Sun's oscillation about the center of mass of the solar system, predicted that cycle 24 will start in May 2006, reach its maximum in October 2011, and end in May 2016. Meyer (1998) predicted that cycle 24 will reach its maximum in 2014 by the model of modulation of amplitude and phase variations.
Wilson $(1987,1993)$ divided all solar cycles into two types, long and short, and found that CorrCoef $=-0.81$ for long-period cycles, -0.86 for short-period cycles (using data of cycles 8-22), and that there is no statistically important relationship among them all. From this bimodal distribution, the extremum times can be predicted (Wilson 1988, 1993; Wilson et al. 1996). However recently, Hathaway \& Wilson (2005) pointed out that this bimodal distribution of cycle length cannot be distinguished from a normal (Gaussian) distribution.

The relationship between $T_{\mathrm{d}}$ and $T_{\mathrm{a}}$ is studied in this paper, using the newly defined extremum times (Du et al. 2006a). We find a statistically important relationship between $T_{\mathrm{d}}$ of a given cycle and $T_{\mathrm{a}}$ of three cycles earlier.

\section{Conventional and weighted average $T_{d}$ and $T_{a}$}

In the present study, we use the 13-month running mean SNs (Gleissberg filter). Monthly $\mathrm{SNs}$ are taken from http://wwW.ngdc.noaa.gov/stp/SOLAR/getdata.html.

An extremum time is conventionally defined as the date (if more than one date have the same extremum value then some mean date is used) of the occurrence of the extremum (Letfus 1994) in the 13-month running mean SNs (Cols. 2, 3 in Table 1). Usually there are several dates when the SNs have the same minimum or maximum value. To determine the extremum times, some authors have used additional parameters, such as first spotless day (Wilson 1995), number of spot groups, 10.7-cm radio flux, total magnetic flux, Ca II K index, He I 1083 equivalent width, total irradiance, or the number of active regions (Harvey \& White 1999, and references therein). Different definitions of the extremum times will make the cycle lengths have a few months' difference (Harvey \& White 1999).

The cycle lengths do not have strong correlation coefficients with other parameters describing solar activity cycles. One reason may be that the SNs often have more than one peak, such as in cycle 23 , and the maximum time is usually defined as that of the highest (e.g., May 2000 in cycle 23). This choice seems 


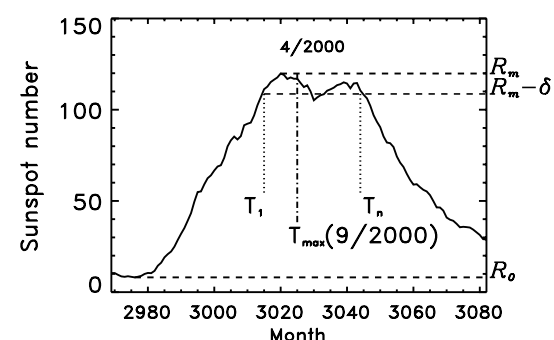

Fig. 1. 13-month running mean SNs (1996-2005), including a maximum $R_{\mathrm{m}}$, where $R_{0}$ is the minimum from the onset to end of this cycle, $\delta=0.1\left(R_{\mathrm{m}}-R_{0}\right), T_{1}$ and $T_{\mathrm{n}}$ the times when $R=R_{\mathrm{m}}-\delta$, and $T_{\max }$ the time of this maximum derived from Eq. (1).

to stress the short rather than long trend behavior. For this reason, we make use of the weighted extremum times by Du et al. (2006a).

Let $R_{\mathrm{m}}$ be the maximum $\mathrm{SN}$ of a given cycle, $R_{0}$ the minimum SN from the onset to end of that cycle, $R_{i}$ the $\mathrm{SN}$ value near this maximum, and $T_{i}$ (from Jan. 1749, in units of months) the corresponding time of $R_{i}$. The value of $R_{i}$ is taken to satisfy the condition: $R_{\mathrm{m}}-\delta \leq R_{i} \leq R_{\mathrm{m}}$ with $\delta=0.1\left(R_{\mathrm{m}}-R_{0}\right)$, and $\mathrm{n}$ is the number of $R_{i}$ values satisfying this condition (Fig. 1).

The maximum time of this cycle is defined as the weighted average time,

$T_{\max }=\frac{1}{\sum_{i=1}^{n} w_{i}} \sum_{i=1}^{n} w_{i} T_{i}$,

where $w_{i}=1 /\left(R_{\mathrm{m}}-R_{i}\right)$ is the weight of $T_{i}$, and the nearer it is to $R_{\mathrm{m}}$, the bigger the weight. When $R_{i}=R_{\mathrm{m}}, w_{i}$ is taken as $3 w^{\prime}$; here, $w^{\prime}$ is the maximum weight for $R_{i} \neq R_{\mathrm{m}}$ in this cycle. The minimum time $\left(T_{\min }\right)$ is defined similarly (Du et al. 2006a). Thus, $T_{\mathrm{d}}$ is defined as the time difference between the maximum and the minimum following it, and $T_{\mathrm{a}}$ the time difference between the maximum and the previous minimum.

The values of $T_{\min }, T_{\max }, T_{\mathrm{a}}$, and $T_{\mathrm{d}}$ are listed in Table 1 for cycles $1-23$. The conventional and weighted average values are separated by a vertical line. The more reliable values since cycle 8 (Eddy 1976) and the less reliable values before cycle 8 are separated by a horizontal line.

With this weighted average definition, the extremum times reflect those of extrema in long trend behavior. The time of an extremum is determined not only by the time of this extremum, but also by the times of $R_{i}$ near this extremum. The mean differences between the weighted average and conventional minmin cycle lengths (period between two successive minima) are about 2.1 and 2.6 months for cycles $1-22$ and 8-22, respectively (see Table 1). The max-max cycle lengths (period between two successive maxima) from the weighted average definition have about 3.3 and 5.4 months mean differences with those from the conventional definition and from the median method (available for cycles 11-22, Mursula \& Ulich 1998), respectively, while the ones from the median method have about 6.5 months mean difference with those from the conventional definition for cycles 11-22.

\section{Results}

\subsection{Relationship between conventional $T_{\mathrm{d}}$ and $T_{\mathrm{a}}$}

To study the relationship between $T_{\mathrm{d}}$ and $T_{\mathrm{a}}$, we have calculated the cross-correlation coefficients (CorrCoef) between $T_{\mathrm{d}}$ and $T_{\mathrm{a}}$
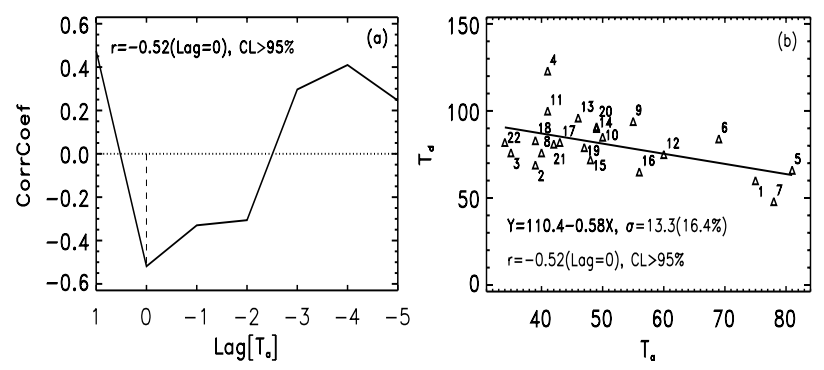

Fig. 2. Cycles 1-22: a) CorrCoefs between conventional $T_{\mathrm{d}}$ and $T_{\mathrm{a}}$ at lags from 1 to -5 , with data of cycles $1-22$. The most significant CorrCoef, -0.52 , is at lag 0, confidence level $>95 \%$. b) Scatter plot of $T_{\mathrm{d}}$ vs. $T_{\mathrm{a}}$ (triangles). The linear regression equation is $T_{\mathrm{d}}=$ $110.4-0.58 T_{\mathrm{a}}$, and the standard error, $\sigma=13.3(16.4 \%)$.
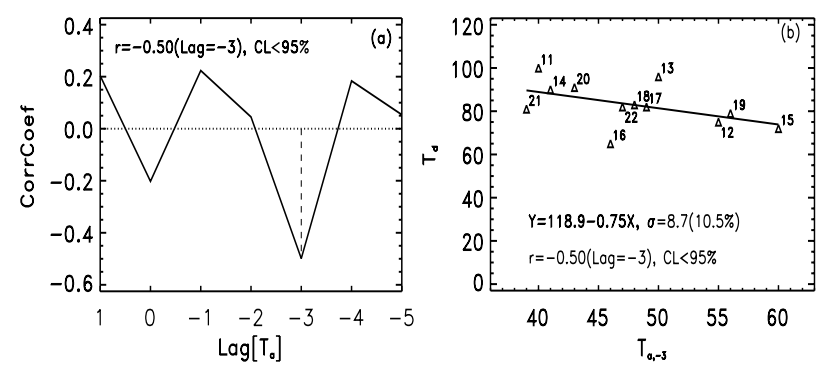

Fig. 3. 8-22: a) CorrCoefs between conventional $T_{\mathrm{d}}$ and $T_{\mathrm{a}}$ at lags from 1 to -5 , with data of cycles $8-22$. The CorrCoef is only -0.19 at lag 0 . The most significant CorrCoef, -0.50 , is now at lag -3 , confidence level $<95 \%$. b) Scatter plot of $T_{\mathrm{d}}$ vs. $T_{\mathrm{a},-3}$. The linear regression equation is $T_{\mathrm{d}}=118.9-0.75 T_{\mathrm{a},-3}$, the standard error, $\sigma=8.7(10.5 \%)$.

at lags (of $T_{\mathrm{a}}$ ) from 1 to -5 , as shown in Fig. $2 \mathrm{a}$, using the conventional definition for cycles $1-22$ (Cols. 4 and 5 in Table 1). The most significant CorrCoef, -0.52 , is at lag 0 , confidence level $>95 \%$. It means that $T_{\mathrm{d}}$ correlates best with $T_{\mathrm{a}}$ of the same cycle. Figure $2 \mathrm{~b}$ illustrates the scatter plot of $T_{\mathrm{d}}$ vs. $T_{\mathrm{a}}$. The linear regression equation is

Cycles $1-22, \operatorname{lag}=0:\left\{\begin{aligned} T_{\mathrm{d}} & =110.4-0.58 T_{\mathrm{a}} \\ \sigma & =13.3(16.4 \%),\end{aligned}\right.$

where $\sigma=13.3$ is the regression standard deviation, and $16.4 \%$ is its relative error. Substituting $T_{\mathrm{a}}=50$ of cycle 23 into Eq. (2), the value of $T_{\mathrm{d}}$ can be estimated as $T_{\mathrm{d}}(23)=81.4 \pm 13.3$ (month).

It should be noted that the CorrCoef $(-0.52)$ is insignificant. The values of $T_{\mathrm{d}}$ are all longer than the corresponding $T_{\mathrm{a}}$ since cycle 8 (Cols. 4 and 5 in Table 1 ), but some values of $T_{\mathrm{d}}$ are shorter than the corresponding $T_{\mathrm{a}}$ for cycles $1-7(1,5,7)$. One reason may be that the SNs before cycle 8 are of a poorer quality than those after cycle 8 (Eddy 1976). Now, we utilize the more reliable data of cycle 8-22, and recalculate the results as shown in Fig. 3.

The CorrCoef between $T_{\mathrm{d}}$ and $T_{\mathrm{a}}$ (at lag 0 ) is only -0.19 . The most significant CorrCoef, -0.50 , is now at lag -3 , confidence level $<95 \%$. The linear regression equation of $T_{\mathrm{d}} \mathrm{vs}$. $T_{\mathrm{a},-3}$ is

Cycles $8-22$, lag $=-3:\left\{\begin{aligned} T_{\mathrm{d}} & =118.9-0.75 T_{\mathrm{a},-3} \\ \sigma & =8.7(10.5 \%),\end{aligned}\right.$

where $T_{\mathrm{a},-3}$ means the value of $T_{\mathrm{a}}$ three cycles earlier. Substituting $T_{\mathrm{a},-3}=52$ of cycle 20 into Eq. (3), the $T_{\mathrm{d}}$ of cycle 23 can be estimated as $T_{\mathrm{d}}(23)=79.9 \pm 8.7$ (month).

Using conventionally defined $T_{\mathrm{d}}$ and $T_{\mathrm{a}}$, the CorrCoefs ( -0.52 and -0.50 for cycles $1-22$ and $8-22$, respectively) are 
Table 1. The values of $T_{\min }, T_{\max }, T_{\mathrm{a}}$, and $T_{\mathrm{d}}$ for cycles $1-23$ from conventional and weighted average definition.

\begin{tabular}{cllrr|llrr}
\hline \hline & \multicolumn{9}{c}{ Conventional } & \multicolumn{5}{c}{ Weighted average } \\
No. & $T_{\min }^{a}$ & $T_{\max }^{a}$ & $T_{\mathrm{a}}$ & $T_{\mathrm{d}}$ & $T_{\min }^{d}$ & $T_{\max }^{d}$ & $T_{\mathrm{a}}$ & $T_{\mathrm{d}}$ \\
& $\mathrm{yr}(\mathrm{mo})$ & $\mathrm{yr}(\mathrm{mo})$ & $\mathrm{mo}$ & $\mathrm{mo}$ & $\mathrm{yr}(\mathrm{mo})$ & $\mathrm{yr}(\mathrm{mo})$ & $\mathrm{mo}$ & $\mathrm{mo}$ \\
\hline 1 & $1755(3)$ & $1761(6)$ & 75 & 60 & $1755(5)$ & $1761(5)$ & 72 & 60 \\
2 & $1766(6)$ & $1769(9)$ & 39 & 69 & $1766(5)$ & $1769(10)$ & 41 & 68 \\
3 & $1775(6)$ & $1778(5)$ & 35 & 76 & $1775(6)$ & $1778(5)$ & 35 & 75 \\
4 & $1784(9)$ & $1788(2)$ & 41 & 123 & $1784(8)$ & $1788(3)$ & 43 & 122 \\
5 & $1798(5)$ & $1805(2)$ & 81 & 66 & $1798(5)$ & $1804(12)$ & 79 & 67 \\
6 & $1810(8)$ & $1816(5)$ & 69 & 84 & $1810(7)$ & $1816(6)$ & 71 & 81 \\
7 & $1823(5)$ & $1829(11)$ & 78 & 48 & $1823(3)$ & $1830(1)$ & 82 & 45 \\
\hline 8 & $1833(11)$ & $1837(3)$ & 40 & 76 & $1833(10)$ & $1837(4)$ & 42 & 75 \\
9 & $1843(7)$ & $1848(2)$ & 55 & 94 & $1843(7)$ & $1848(4)$ & 57 & 93 \\
10 & $1855(12)$ & $1860(2)$ & 50 & 85 & $1856(1)$ & $1860(2)$ & 49 & 85 \\
11 & $1867(3)$ & $1870(8)$ & 41 & 100 & $1867(3)$ & $1870(10)$ & 43 & 96 \\
12 & $1878(12)$ & $1883(12)$ & 60 & 75 & $1878(10)$ & $1883(12)$ & 62 & 64 \\
13 & $1890(3)$ & $1894(1)$ & 46 & 96 & $1889(4)$ & $1893(12)$ & 56 & 94 \\
14 & $1902(1)$ & $1906(2)$ & 49 & 90 & $1901(10)$ & $1906(4)$ & 54 & 87 \\
15 & $1913(8)$ & $1917(8)$ & 48 & 72 & $1913(7)$ & $1917(9)$ & 50 & 70 \\
16 & $1923(8)$ & $1928(4)$ & 56 & 65 & $1923(7)$ & $1928(3)$ & 56 & 66 \\
17 & $1933(9)$ & $1937(4)$ & 43 & 82 & $1933(9)$ & $1937(6)$ & 45 & 81 \\
18 & $1944(2)$ & $1947(5)$ & 39 & 83 & $1944(3)$ & $1948(3)$ & 48 & 73 \\
19 & $1954(4)$ & $1958(3)$ & 47 & 79 & $1954(4)$ & $1958(2)$ & 46 & 80 \\
20 & $1964(10)$ & $1968(11)$ & 49 & 91 & $1964(10)$ & $1969(2)$ & 52 & 87 \\
21 & $1976(6)$ & $1979(12)$ & 42 & 81 & $1976(5)$ & $1979(12)$ & 43 & 77 \\
22 & $1986(9)$ & $1989(7)$ & 34 & 82 & $1986(5)$ & $1989(7)$ & 38 & 84 \\
23 & $1996(5)^{b}$ & $2000(5)^{c}$ & 48 & & $1996(7)$ & $2000(9)$ & 50 & $(78)^{e}$ \\
\hline
\end{tabular}

$a$ The values with conventional definitions are taken from Letfus (1994), except for cycle 23 .

${ }^{b}$ This date is taken from Li et al. (2001).

${ }^{c}$ This date is taken from http://wWW.ngdc.noaa.gov/stp/SOLAR/ getdata.html.

${ }^{d}$ The values with weighted average definition are taken from Eq. (1) and the alike.

${ }^{e}$ Predicted value from Eq. (6).

low, and the confidence levels ( $<99 \%$ and $<95 \%$ for cycles $1-22$ and $8-22$, respectively) are low, and the confidence levels $(<99 \%$ and $<95 \%$ for cycles $1-22$ and 8-22, respectively) are not high. If we take $T_{\mathrm{d}}(23)=81 \pm 13$ months extrapolated from Eq. (2) as an estimated value of the descending phase duration in cycle 23 with the confidence level greater than $95 \%$, then the onset of cycle 24 should be in February $2007 \pm 9$ months.

\subsection{Correlation between weighted average $T_{d}$ and $T_{a}$ for cycles 1-22}

Now we study the relationship between the newly defined $T_{\mathrm{d}}$ and $T_{\mathrm{a}}$ of cycles 1-22 (Cols. 8 and 9 in Table 1). The results are shown in Fig. 4. The most significant CorrCoef is 0.54, at lag -4 , confidence level $>95 \%$. The linear regression equation of $T_{\mathrm{d}}$ vs. $T_{\mathrm{a},-4}$ is

Cycles $1-22, \operatorname{lag}=-4:\left\{\begin{aligned} T_{\mathrm{d}} & =50.9+0.50 T_{\mathrm{a},-4} \\ \sigma & =10.7(13.7 \%),\end{aligned}\right.$

where $T_{\mathrm{a},-4}$ means the value of $T_{\mathrm{a}}$ four cycles earlier. Substituting $T_{\mathrm{a},-4}=46$ of cycle 19 into Eq. (4), the value of $T_{\mathrm{d}}$ can be estimated to be $T_{\mathrm{d}}(23)=73.9 \pm 10.7$ (month).

For a comparison with Fig. $2 b$, we illustrate the scatter plot of $T_{\mathrm{d}}$ vs. $T_{\mathrm{a}}$ (of lag 0 ) in Fig. 5. The linear regression equation is

Cycles $1-22, \operatorname{lag}=0:\left\{\begin{aligned} T_{\mathrm{d}} & =109.4-0.58 T_{\mathrm{a}} \\ \sigma & =13.6(17.2 \%) .\end{aligned}\right.$

It is similar to Eq. (2). The CorrCoef is -0.49 (at lag 0), confidence level $>95 \%$. Substituting $T_{\mathrm{a}}=50$ of cycle 23 into Eq. (5), we can predict $T_{\mathrm{d}}(23)=80.4 \pm 13.6$ (month).
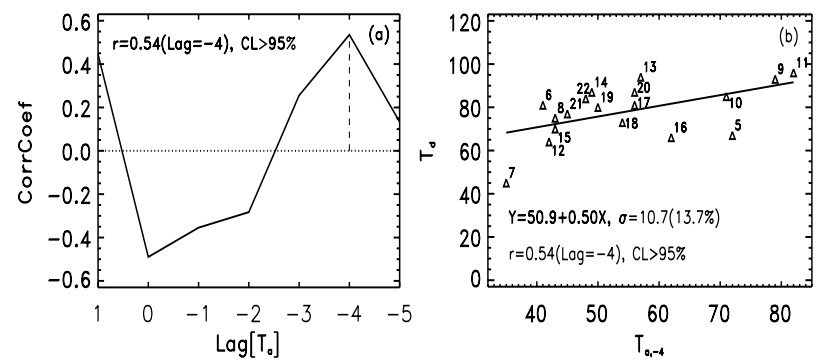

Fig. 4. 1-22: a) CorrCoefs between weighted average $T_{\mathrm{d}}$ and $T_{\mathrm{a}}$ at lags from 1 to -5 , with data of cycles $1-22$. The CorrCoef is only -0.49 at lag 0, confidence level $>95 \%$. The most significant CorrCoef is 0.54 , at lag -4 , confidence level $>95 \%$. b) Scatter plot of $T_{\mathrm{d}}$ vs. $T_{\mathrm{a},-4}$. The linear regression equation is $T_{\mathrm{d}}=50.9+0.50 T_{\mathrm{a},-4}$, the standard error, $\sigma=10.7(13.7 \%)$.

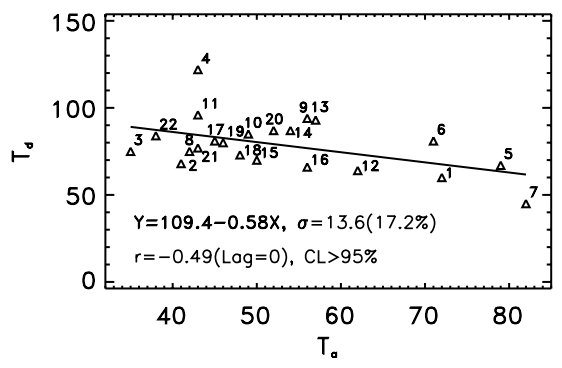

Fig. 5. Scatter plot of $T_{\mathrm{d}}$ vs. $T_{\mathrm{a}}$ at lag 0 . The CorrCoef is -0.49 with confidence level $>95 \%$. The linear regression equation is $T_{\mathrm{d}}=109.4-$ $0.58 T_{\mathrm{a}}$, the standard error is $\sigma=13.6(17.2 \%)$.
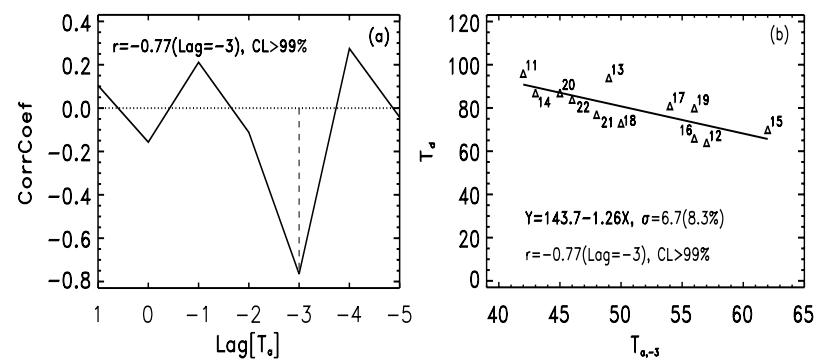

Fig. 6. 8-22: a) CorrCoefs between weighted average $T_{\mathrm{d}}$ and $T_{\mathrm{a}}$ at lags from 1 to -5 , with data of cycles 8-22. The CorrCoef between $T_{\mathrm{d}}$ and $T_{\mathrm{a}}$ (at lag 0) is only -0.16 . The significant CorrCoef is -0.77 , at lag -3 , confidence level $>99 \%$. b) Scatter plot of $T_{\mathrm{d}}$ vs. $T_{\mathrm{a},-3}$. The linear regression equation is $T_{\mathrm{d}}=143.7-1.26 T_{\mathrm{a},-3}$, the standard error, $\sigma=$ $6.7(8.3 \%)$.

For cycles $1-22$, although the CorrCoef for lag $=0(-0.49)$ is a little worse than that for lag $=-4(0.54)$, both are statistically insignificant.

\subsection{Correlation between weighted average $T_{d}$ and $T_{a}$ for cycles 8-22}

For cycles $1-22$, although the CorrCoef for lag $=0(-0.49)$ is a little worse than that for lag $=-4(0.54)$, both are statistically insignificant. Now, we utilize the more reliable data of cycles 8-22 to reexamine the results, as shown in Fig. 6.

The CorrCoef between $T_{\mathrm{d}}$ and $T_{\mathrm{a}}$ (at lag 0 ) is only -0.16 . The significant CorrCoef is -0.77 , at lag -3 , confidence level $>99 \%$. Figure $6 \mathrm{~b}$ illustrates the scatter plot of $T_{\mathrm{d}}$ vs. $T_{\mathrm{a},-3}$, and the linear regression equation is given by

Cycles $8-22, \operatorname{lag}=-3:\left\{\begin{aligned} T_{\mathrm{d}} & =143.7-1.26 T_{\mathrm{a},-3} \\ \sigma & =6.7(8.3 \%),\end{aligned}\right.$ 
where $T_{\mathrm{a},-3}$ means the value of $T_{\mathrm{a}}$ three cycles earlier. It implies that $T_{\mathrm{d}}$ correlates best with $T_{\mathrm{a}}$ three cycles earlier. This threecycle effect in Fig. 6a is similar to that in Fig. 3a, but has a higher CorrCoef.

Substituting $T_{\mathrm{a},-3}=52$ of cycle 20 into Eq. (6), the $T_{\mathrm{d}}$ in the current cycle can be estimated as $T_{\mathrm{d}}(23)=78.2 \pm 6.7$ (month). It is slightly shorter than $81.4 \pm 13.3,79.9 \pm 8.7$, and $80.4 \pm 13.6$ from Eqs. (2), (3), and (5), respectively, and longer than $73.9 \pm$ 10.7 from Eq. (4). If we take the descending phase duration of cycle 23 as $78.2 \pm 6.7$ (month) for the higher CorrCoef $(-0.77)$, then the onset of cycle 24 should be in March $2007 \pm 7$ months.

The CorrCoef, -0.77 , between $T_{\mathrm{d}}$ and $T_{\mathrm{a},-3}$ is much more significant than all other cases (about \pm 0.5 ), either with other lags or due to the conventional definition (Figs. 2-6). And the confidence level $(>99 \%)$ is also higher than other cases. This three-cycle effect is similar to that in the relationship between the maxima and cycle lengths: maxima correlate best with cycle lengths three cycles earlier (Silverman 1992; Orfila et al. 2002; Solanki el al. 2002; Du et al. 2006b).

\section{Discussions and conclusions}

The times of both the maxima and minima of sunspot numbers are important parameters in solar activity cycles. It is usually believed that $R_{\mathrm{m}}$ has a high correlation coefficient with $T_{\mathrm{a}}$, but a low one with $T_{\mathrm{d}}$ (Waldmeier 1935, 1939; Wilson 1993; Usoskin $\&$ Mursula 2003). The method most often used to predict the maximum times is probably the relationship between $R_{\mathrm{m}}$ and $T_{\mathrm{a}}$ (Gleissberg 1971; Wilson 1988, 1993). Conventionally, the $T_{\mathrm{d}}$ does not have a high correlation coefficient either with $R_{\mathrm{m}}$ or with other parameters, such as $T_{\mathrm{a}}$ (Usoskin \& Mursula 2003). The correlation coefficient between $T_{\mathrm{d}}$ and $T_{\mathrm{a}}$ is only about -0.5 . The high correlation coefficient between $T_{\mathrm{d}}$ and $T_{\mathrm{a}}$ exists only for the bimodal distribution of cycle length (Wilson 1987), but Hathaway \& Wilson (2005) pointed out that this bimodal distribution cannot be distinguished from a normal (Gaussian) distribution.

With the newly defined weighted average extremum times, $T_{\mathrm{d}}$ is found to be well correlated (CorrCoef $=-0.77$ ) with $T_{\mathrm{a}}$ three cycles earlier at confidence level greater than $99 \%$, using the more reliable data since cycle 8 . It is similar to the three-cycle effect in the relationship between $R_{\mathrm{m}}$ and cycle length (Silverman 1992; Orfila et al. 2002; Solanki el al. 2002; Du et al. 2006b; Du 2006b), whereas it is insignificant (CorrCoef $=-0.50$ ) for the corresponding correlation between the conventional $T_{\mathrm{d}}$ and $T_{\mathrm{a}}$ at lag -3 with the same period data since cycle 8 . It implies that the newly defined extremum times reflect those in the long-term behavior of sunspot numbers rather than those of the highest or lowest values.

The maximum time in cycle 23 is in September 2000 from the new definition, four months later than the conventional one (May 2000). The onset of cycle 24 should be in March $2007 \pm$ 7 months with our technique. It is near to May 2007 according to Hanslmeier et al. (1999), later than May 2006 according to Landscheidt (1999), and later than or near to January 2006 May 2007 according to Wilson (1998). Incidentally, it is also close to the date, February $2007 \pm 13$ months, predicted from the relationship between the conventional $T_{\mathrm{d}}$ and $T_{\mathrm{a}}$.
From the analysis above, our main conclusions are as follows,

1. The weighted average definition of the extremum times stresses the property of whole cycle structure rather than that of the highest or lowest one. With this definition, each cycle is treated as having only one main peak, even if it has multiple peaks, such as in cycles 22-23.

2. The descending phase durations are well correlated (CorrCoef $=-0.77$ ) with the ascending phase durations three cycles earlier, with confidence level greater than $99 \%$, using the more reliable data of cycles 8-22.

3. The descending phase duration of cycle 23 is predicted to be $78 \pm 7$ months, and then the next cycle (24) should start near March $2007 \pm 7$ months.

Acknowledgements. The author is grateful to the anonymous referee for helpful comments and English improvements. We express our special acknowledgements to Professor T. Kiang for linguistic improvements. This work is supported by NSFC through grant 10233050, and Chinese Academy of Sciences through grant KGCX 2-SW-408.

\section{References}

Du, Z. L. 2006a, New Astron., in press

Du, Z. L. 2006b, AJ, accepted

Du, Z. L., Wang, H. N., \& He, X. T. 2006a, Chin. J. Astron. Astrophys., 6(3), 338

Du, Z. L., Wang, H. N., \& He, X. T. 2006b, Chin. J. Astron. Astrophys., in press Eddy, J. A. 1976, Science, 192, 1189

Gleissberg, W. 1971, Sol. Phys., 21, 240

Hanslmeie, A., Denkmayr, K., \& Weiss, P. 1999, Sol. Phys., 184, 213

Harvey, K. L., \& White, O. R. 1999, J. Geophys. Res., 104, 19759

Hathaway, D. H., \& Wilson, R. M. 2005, Sol. Phys., 224, 19

Kane, R. P. 1978, Nature, 274, 139

Kane, R. P. 2001, Sol. Phys., 202, 359

Landscheidt, T. 1999, Sol. Phys., 189, 415

Letfus, V. 1994, Sol. Phys., 149, 405

Li, K. J., Yun, H. S., \& Gu, X. M. 2001, A\&A, 368, 285

Meyer, F. D. 1998, Sol. Phys., 181, 201

Mursula, K., \& Ulich, Th. 1998, Geophys. Res. Lett., 25, 1837

Ohl, A. I. 1966, Soln. Dann No. 9, 73

Orfila, A., Ballester, J. L., Oliver, R., Alvarez, A., \& Tintore, J. 2002, A\&A, 386, 313

Silverman, S. M. 1992, Rev. Geophys., 30, 333

Solanki, S. K., Krivova, N. A., Schussler, M., \& Fligge, M. 2002, A\&A, 396, 1029

Thompson, R. J. 1993, Sol. Phys., 148, 383

Usoskin, I. G., \& Mursula, K. 2003, Sol. Phys., 218, 319

Waldmeier, M. 1935, Astron. Mitt. Zrich, 14(133), 105

Waldmeier, M. 1939, Astron. Mitt. Zrich, 14, (138), 439

Wang, J. L., \& Han, Y. B. 2000, Chin. J. Space Science, 20, 3

Wang, J. L, Gong, J. C., Liu, S. Q., Le, G. M., \& Sun, J. L. 2002, Chin. J. Astron. Astrophys., 2, 557

Whitehouse, D. R. 1985, A\&A, 145, 451

Wilson, R. M. 1987, Sol. Phys., 108, 195

Wilson, R. M. 1988, J. Geophys. Res., 93, 10011

Wilson, R. M. 1990, Sol. Phys., 125, 143

Wilson, R. M. 1993, J. Geophys. Res., 98, 1333

Wilson, R. M. 1995, Sol. Phys., 158, 197

Wilson, R. M. 1998, J. Geophys. Res., 103, 11159

Wilson, R. M., Hathaway, D. H., \& Reichmann, E. J. 1996, J. Geophys. Res., 101,19967

Wilson, R. M., Hathaway, D. H., \& Reichmann, E. J. 1998, J. Geophys. Res., 103,6595

Zhang, G. Q., \& Wang, H. N. 1999, Sol. Phys., 188, 391 